\title{
A Study on the Success Cases about AI RPA (Robotic Process Automation) in Manufacturing Industry
}

\author{
JeongBeom Kim \\ Head Professor of BigdataAI Dept., Namseoul University, Daehak Ro, Sunghwaneup, \\ Choongnam, Korea \\ jbkim@nsu.ac.kr
}

\begin{abstract}
The main purpose of this paper is to study success cases about AI RPA (Robotic Process Automation) focus on the manufacturing industry. By implementing robotic process automation (RPA), an artificial intelligence solution at the manufacturing site, differentiation strategies such as maintenance of manufacturing systems, process innovation and efficiency, quality improvement, global collaboration system, and simulation of manufacturing business can be realized. Recently, the introduction of artificial intelligence technology that can reduce the manufacturing cost by building BPA around advanced manufacturing companies has been increasing the success of winter innovation. BPA technology is making use of PLM (Product Lifecycle Management) data at manufacturing sites. Data of PLM System, a framework of manufacturing sites, has integrated data that manages all phases of a product's entire life cycle, from development ideas to disposal, so that the product's lifecycle is based on three dimensions. In addition, the design is digitally processed, the prototypes are digitally verified, the production processes and production methods are digitally verified, and monitoring and simulation are supported. We will study how to differentiate the competitiveness of manufacturers through the case of building BPA, an artificial intelligence technology, in the manufacturing field.
\end{abstract}

Keywords: AI, RPA, PLM, Manufacturing industry, Success case

\section{Introduction}

This study presents successful cases of improving efficiency and competitive advantage of companies through successful cases in the manufacturing site of BPA technology. The main purpose of building BPA technology in the manufacturing sites is to apply BPA to automate tasks that require cognitive ability, such as paper document numbering, security-enhanced site linkage, and inspection of various documents. As the figure below shows in the historical flow of object detection, image recognition technology plays a pivotal role in the core of BPA technology manufacturing site construction.

\section{Related study}

RPA AI is so popular that it is the hottest new technology of AI these days. RPA is expanding its business mainly from processing unstructured data to processing unstructured data by adding

Article history:

Received (April 27, 2019), Review Result (June 19, 2019), Accepted (September 26, 2019) 
artificial intelligence. RPA AI success stories from leading domestic and international companies provide insight into how the RPA is now delivering digital workforce and how it will evolve in the future. Robotic Process Automation benefits the Enterprise with many success cases. It is getting more and more spreading. The digital workforce of the future is not about robots replacing 100\% of human work but about the collaboration of man and robot. RPA is moving to IPA (Intelligent Process Automation) to take over difficult and sensitive works. Follow picture show RPA image recognition history [1][2].

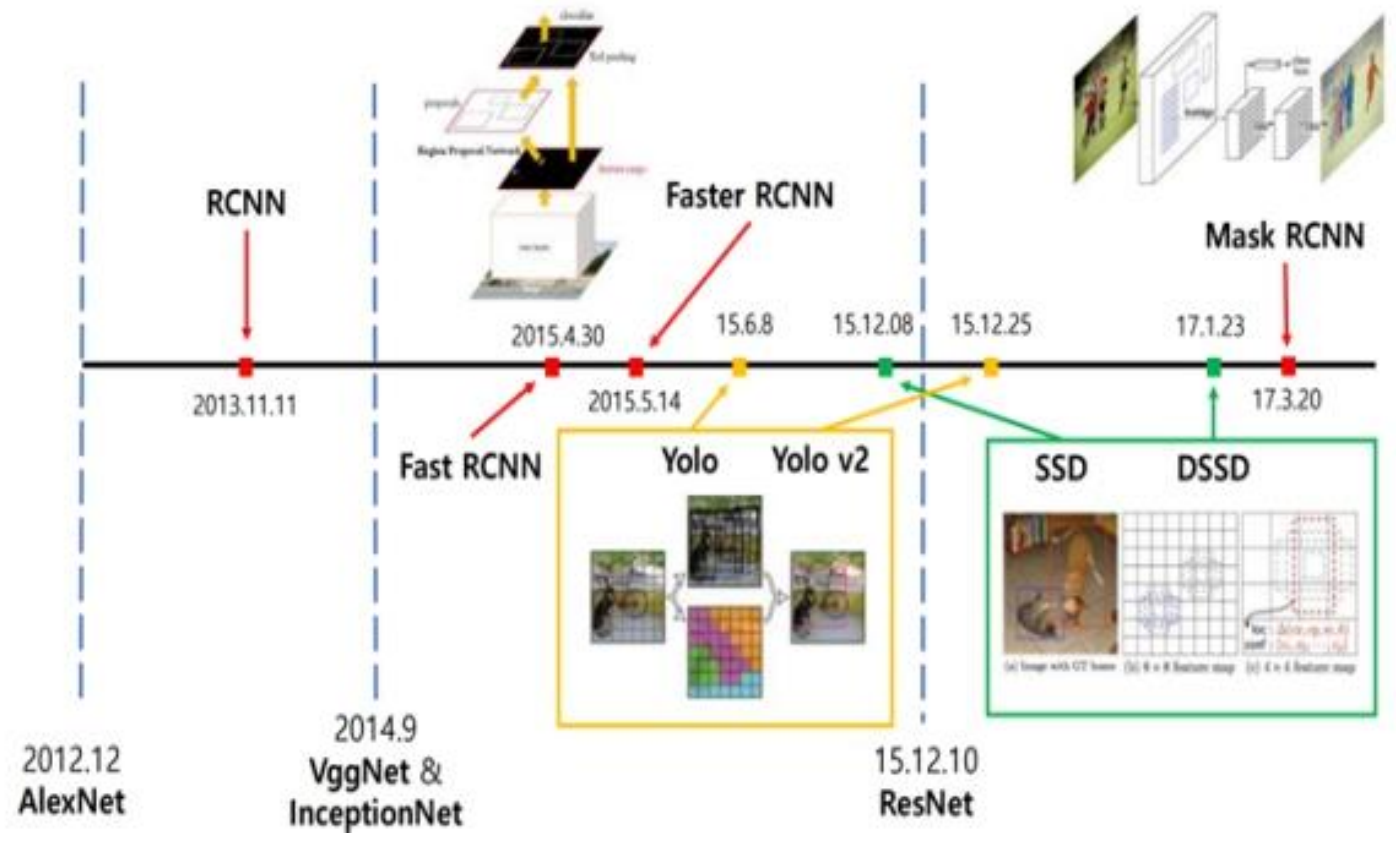

Figure 1. RPA image recognition history

\section{RPA frame work}

The RPA Framework can be summarized as follows. First, based on image recognition, the task is fully automated in terms of universal non-standard GUI. Second, based on user behavior, it can quickly and easily deliver the contents of the task to the software robot. Third, it enables business automation by interworking PC and mobile terminal by relaying data between terminals. Fourth, it enables automation of security-enhancing sites of companies including public institutions. Fifth, it analyzes the quality of work performed by software robots and makes it possible to inquire about the results in detail. It also works closely with the PLM system, the heart of the manufacturing site. In manufacturing site, AI RPA has been used to automate the tasks. Based on the RPA OCR (Object Character Recognition) technology, we can identify some examples of automating non-regular paperwork in manufacturing industry [2].

\subsection{Early warning model}

An analytical model that uses quality assurance claims (Claim) information, the most practical information that shows that a product purchased during the warranty period after the product launch is up and running. Based on this, it is a predictive model that suggests appropriate alert days by analyzing patterns and identifying trends of defective rates through statistical analysis techniques to predict when to surge. 


\subsection{Detective cause analysis model}

In various manufacturing fields, it is very important to find the process line where defect occurs in the production stage. To this end, the main factors influencing the defective process are searched based on product production/shipment information, defect occurrence information, and process line information, and the correlation with the defect information is analyzed by decision tree method. The model that identifies the cause of occurrence.

\subsection{Critical success factors for RPA implementation}

For the successful RPA implementation, there are five key success factors. First one is collect data from internal and external source continuously. The second one is to secure the quality of data. The third one is to establish hardware system which is reliable, available and ease to use. The fourth one is to secure the AI expert and organization to support RPA system. The last one is to train end users effectively. For example, RPA system is being applied in call center to increase customer satisfaction and competitiveness. Following diagram shows the flow of natural language process which is one of the core technologies of RPA.

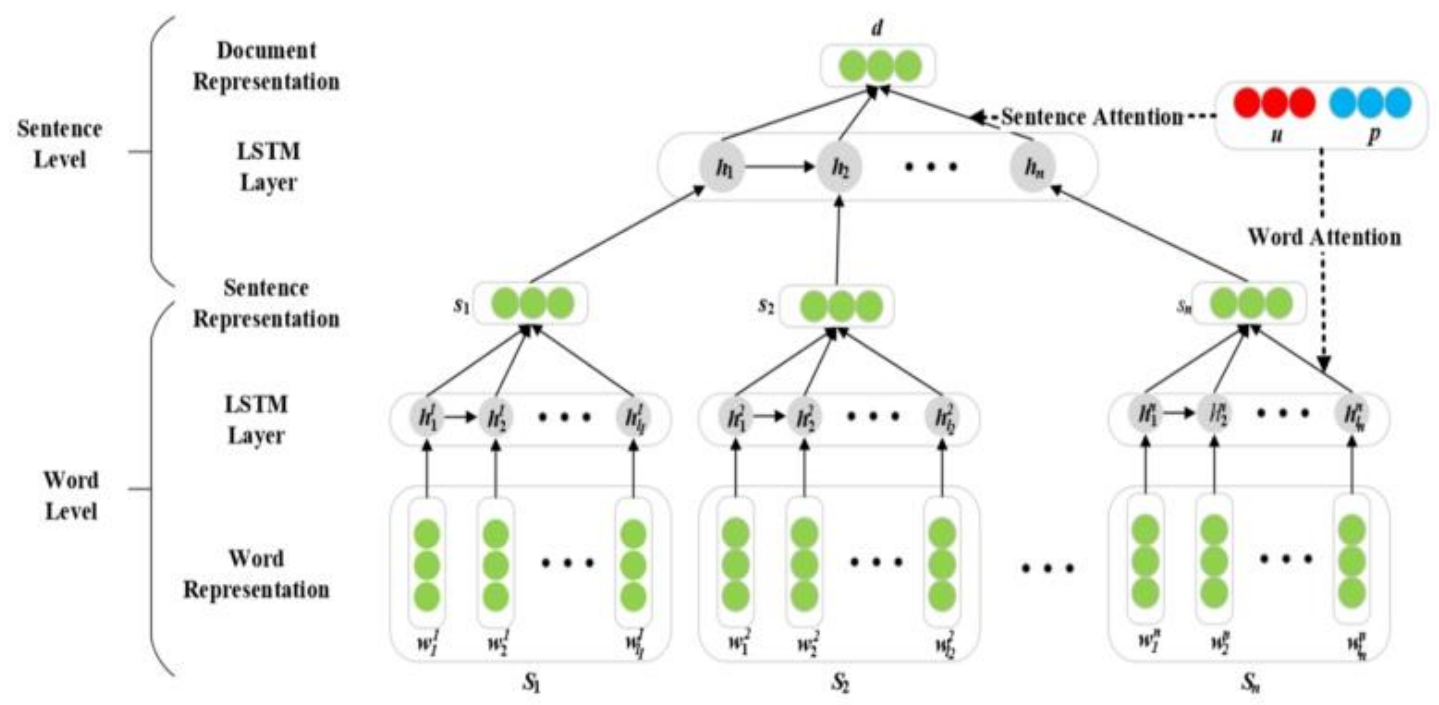

Figure 2. Flow of RPA natural language process

\subsection{Success story of RPA implementation}

As a result of the RPA implementation, finance RPA is being used for accuracy, sales RPA is being applied for efficiency, production RPA is being used for time savings. Competitiveness was also achieved by accumulating data. RPA automates cost-related numerical tasks, such as rate entry, to reduce typing errors with accuracy and automating the checking and processing of deposit accounts.

The result is more customer encounters and increased sales opportunities. Export tariff refunds and purchase slips processed at the end of the year are automated, allowing timely closing. Overnight work and weekend work that was repeated at the end of the month disappeared. Based on the accumulated data, it is possible to purchase through cost prediction and analysis. RPA can ease the burden on business people for everyday tasks and simple repetitive tasks. RPA achieved cost-effectiveness, increased job satisfaction, and reduced 
overtime and delays. The following picture shows the example flow of RPA system $[3][4][5][6][7][8][9][10]$.

\begin{tabular}{|l||l||l||l||l||l|}
\hline Data Analysis & Feature Selection & Supervised & Unsupervised & Deep Learning \\
Pre-processing \\
Collinearity Analysis \\
Statistical Analysis \\
Outlier Analysis \\
Timeseries Analysis \\
Cluster Analysis
\end{tabular}

Figure 3. Example flow of RPA system

\subsection{Case of RPA implementation in semiconductor industry}

Complex semiconductor yield is also the era of cloud and AI analysis. Without RPA there is no AI. In human terms, AI is the brain, and business applications such as ERP, SCM, PLM, and CRM are the limbs. The neural network that connects the brain and limbs is RPA. AI learns from input data, and in the future, companies will analyze data organized through RPA as AI. As a result, companies cannot successfully implement AI without RPA. One of the most important part of RPA system is maintaining big data to train with enough volume to make RPA system accurately. The following picture shows about RPA big data train flow [11][12][13].



Figure 4. Example flow of RPA data train flow 


\section{Performance objective for RPA implementation in manufacturing industry}

RPA objective in manufacturing industry is to develop and deploy smart manufacturing systems with improved performance, collaboration, agility, and ease of integration. The first one is the performance framework for RPA system. This objective is for evaluating and guaranteeing the performance of an RPA system, which are mobility and stability. The goal is to develop methodologies and tools to characterize and combine performance. Second one is the performance of collaborative systems for developing test methods, protocols and information models to evaluate and ensure robot-robot or robot-human collaboration capabilities to complete the mission of the RPA system. The third one is the agility performance of system to develop agility-related metrics, information models, test methods, protocols and test results so that RPA systems can be reconfigured and reworked quickly and easily.[1][14][15][16][17]

\section{Conclusion}

In this study, we proposed a method of making manufacturing innovation by applying AI RPA, which is an artificial intelligence technology, based on big data analysis method and machine learning at the manufacturing site. Algorithm development based on machine learning can achieve the maximum effect through minimum resource input and capitalize its own methodology compared to the manual methodology that calculates and inputs various cases in terms of technology utilization. This is an example of a large domestic company that introduced RPA. In the manufacturing site, AI RPA system are being used to automate the tasks mentioned in this paper. Based on the RPA Object Character Recognition technology, we identified some examples of automating non-regular paperwork. Users 'demands are moving from RPA to IPA. Most of them are planning to expand their AI-based automation tasks. In the future, AI RPA and IPA technology will have a big impact on business mobilization for digital transformation in manufacturing industry to increase competitive power.

\section{References}

[1] KIAT, "Research Trend of Smart Manufacturing in U.S.A," KIAT Industrial Technology Policy Brief, 201421, (2014)

[2] Gridone Ltd., "News Letter," (2019)

[3] C. D. Scott and R. E. Smalley, "Diagnostic ultrasound: Principles and instruments," Journal of Nanosci. Nanotechnology., vol.3, no.2, pp.75-80, (2003)

[4] Smith, T.F. and Waterman, M.S., "Identification of common molecular subsequences,". J. Mol. Biol., vol.147, pp.195-197, (1981)

[5] Michael Negnevitsky, “Artificial intelligence,” Addison Welsley, (2017)

[6] H. S. Nalwa and Editor, "Magnetic nanostructures," American Scientific Publishers, Los Angeles, (2003)

[7] Big Data Computing Technology, Hanbit Academy, pp.14-31, (2016)

[8] IBM project management report, New York, (2004)

[9] H. V. Jansen, N. R. Tas, and J. W. Berenschot, "Encyclopedia of nanoscience and nanotechnology," Edited H. S. Nalwa, American Scientific Publishers, Los Angeles, vol.5, pp.163-275, (2004)

[10] Big Data Computing Technology, Hanbit Academy, pp.14-31, (2016)

[11] OkGi Kim, "Data science,” Ezies Publishing Co, pp.52-55, (2017)

[12] J. Kimura and H. Shibasaki, "Recent advances in clinical neurophysiology," Proceedings of the 10th International Congress of EMG and Clinical Neurophysiology, Kyoto, Japan, October 15-19, (1995) 
[13] World Economic Forum 1, "Big data, big impact: New possibilities for international development," VITAL WAVE CONSULTING, (2012)

[14] H. V. Jansen, N. R. Tas, and J. W. Berenschot, "Encyclopedia of Nanoscience and Nanotechnology", Edited H. S. Nalwa, American Scientific Publishers, Los Angeles, vol.5, pp.163-275, (2004)

[15] OkGi Kim, Data Science, Ezies Publishing Co, pp.52-55, (2017)

[16] D. Allen, "When axioms collide: An unfulfilled opportunity to advance knowledge for man and machine through automated reasoning", International Conference on Computer Systems and Communication Technology, Shenzhen, China, (In Press. Proceedings to be published by Springer), Aug.7-9, (2016)

[17] Landauer T. K., Foltz P. W., and Laham D., "Introduction to Latent Semantic Analysis", Discourse Processes, Vol.25, pp.259-284, (1998)

\section{Authors}

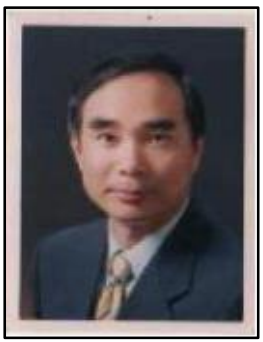

\section{JB(Jeongbeom) Kim}

Head Professor of BigdataAI Department, Namseoul University. $<$ Education >

Seoul National University, majored in Physics B.S.

Yonsei Graduate University, MBA

Soongsil Graduate University, Ph.D

$<$ Work Experience> IBM Korea. SAP Korea 